\title{
El papel de la comunicación en el crowdfunding: estudio de un caso de éxito cool
}

\section{The role of communication in crowdfunding: a case study of cool success}

\author{
Cristina Olarte-Pascual \\ Docente e investigadora \\ (Universidad de La Rioja) \\ Yolanda Sierra-Murillo \\ Docente e investigadora \\ (Universidad de La Rioja) \\ Carmen Ortega \\ Graduada en Administración y Dirección de Empresas \\ (Universidad de La Rioja)
}

Fecha de recepción: 9 de mayo de 2016

Fecha de revisión: 24 de junio de 2016

Para citar este artículo: Olarte-Pascual, C., Sierra-Murillo, Y. y Ortega, C. (2016): El papel de la comunicación en el crowdfunding: estudio de un caso de éxito cool, Icono 14, volumen 14 (2), pp. 280-303. doi: 10.7195/ri14. v14i2.968 


\section{Resumen}

El desarrollo de Internet ha permitido que muchos emprendedores acudan a nuevas fuentes de financiación como el crowdfunding. El objetivo de este artículo es analizar el papel de la comunicación en la captación de fondos. Para ello se comparan dos campañas desarrolladas para un mismo proyecto: una primera, "The Coolest", que no consiguió financiación y otra posterior, "The Coolest Cooler", que recaudó 13.285.226 dólares batiendo un récord de captación de fondos. Los resultados muestran importantes diferencias en la utilización de las siguientes herramientas de persuasión: egocentrismo, tangibilidad, estímulos visuales, emoción, principio, estructura y final del mensaje. Todos los elementos del mensaje deben generar confianza y promover a la acción: participar y financiar el proyecto propuesto. Este trabajo tiene implicaciones prácticas y teóricas al demostrar que la comunicación se convierte en una pieza clave en el éxito de las campañas de crowdfunding.

Palabras clave: Crowdfunding - Comunicación - Crowdfunding de recompensas Persuasion - The Coolest - The Coolest Cooler

\section{Abstract}

The development of the Internet has allowed many entrepreneurs to obtain funding from new sources such as crowdfunding. The aim of this paper is to analyze the role of communication in fundraising. With that purpose, two campaigns developed for the same project were compared. The first one, called "The Coolest", which did not obtain funding, and a later one, "The Coolest Cooler", which by collecting 13.285.226 USD beated a fundraising record. The results show significant differences in the use of the following tools of persuasion: egocentricity, tangibility, visual stimuli, excitement, and the beginning and the end of the message, as well as its structure. All elements of the message should build trust and promote action: participate and finance the proposed project. This work has practical and theoretical implications demonstrating that communication is a key element in the success of the crowdfunding campaigns.

Key Words: Crowdfunding - Communication - Persuasion - Reward crowdfunding The Coolest - The Coolest Cooler 
El papel de la comunicación en el crowdfunding... $\mid 282$

MONOGRÁFICO

\section{Introducción}

El crowdfunding es una forma colectiva, participativa y colaborativa de recaudar fondos que otorga a las multitudes, crowd, el poder de decidir qué proyectos se van a materializar por medio de sus aportaciones monetarias (Roig-Telo, Sánchez-Navarro \& Leibovitz, 2012; Cetti \& Corso, 2014). La Unión Europea define el crowdfunding como una llamada abierta al público para recaudar fondos con un objetivo específico (SpaceTec Capital Partners GmbH, 2014). En el crowdfunding, el público deja de tener un rol pasivo para convertirse en mecenas, en financiador con influencia sobre los productos y procesos de producción ${ }^{1}$. Al compartirse el proceso financiero con el público, aumenta la transparencia $\mathrm{y}$, con ella, la confianza en el proyecto y en sus creadores (Llorca, 2010). En consecuencia y dadas las características comentadas, la forma de comunicar el proyecto pasa a ser un elemento clave del éxito de las campañas de crowdfunding.

Esta forma de financiación colectiva o micromecenazgo se venía usando desde siglos atrás y, por ejemplo, en 1713 sirvió para la traducción de textos religiosos; posteriormente se usó para costear grandes construcciones como el pedestal de la Estatua de la Libertad en 1885 (Kazmark, 2013). En 1997 se llevó a cabo la primera campaña de crowdfunding por Internet y en 2003 se puso en funcionamiento la primera plataforma online, ArtistShare (Sawers, 2014; SpaceTec Capital Partners $\mathrm{GmbH}$, 2014).

El interés y peso del crowdfunding como fuente de financiación se ha potenciado gracias a la generalización del uso de Internet (Lee, DeWester \& Park, 2008) y al aumento del número de plataformas virtuales de crowdfunding (Martínez Gallardo \& Alberich Pascual, 2013; Sánchez-González \& Palomo-Torres, 2015). Dichas plataformas actúan como intermediarios entre los creadores de proyectos y sus posibles financiadores a cambio de una comisión (Asociación Española de Crowdfunding, 2014; Coronado Ruiz \& Larrañaga Rubio; 2015). Además, el desarrollo de las redes sociales ha favorecido su difusión.

En el ámbito académico, si bien se han realizado interesantes trabajos en el campo de la creación cultural (ej. Roig Telo, Sánchez-Navarro \& Leibovitz, 2012; 


\section{MONOGRÁFICO}

Solera-Navarro \& Caballero Fernández-Delgado, 2015), pocos trabajos han analizado las características del mensaje elaborado para presentar los proyectos de crowdfunding en las plataformas de Internet. Este artículo contribuye a llenar este hueco identificado en la literatura y se propone como objetivo analizar el papel de la comunicación en la captación de fondos. Para ello se realiza un estudio comparativo de los mensajes desarrollados en dos campañas ejecutadas para un mismo proyecto. Dichas campañas se emiten en dos momentos diferentes del tiempo y obtienen muy distintos resultados: la primera fracasa y la segunda bate record de recaudación financiera.

El crowdfunding ha crecido en los últimos años y se espera que este crecimiento continúe en los venideros (Crowdfundinsider, 2015; Google Trends, 2015; Dushnitsky et al., 2016). El mercado europeo pasó de recaudar 487 millones de euros en 2012 a 2.957 en 2014 (Figura 1), un crecimiento medio interanual del 146\% (Wardrop et al., 2015). En España, esta financiación alternativa consiguió recaudar 62 millones de euros en 2014, lo que supone un crecimiento del $152 \%$ respecto al año 2012. Estos datos sitúan a España en la sexta posición a nivel europeo por volumen de recaudación, por detrás de Gran Bretaña, Francia, Alemania, Suecia y Holanda.

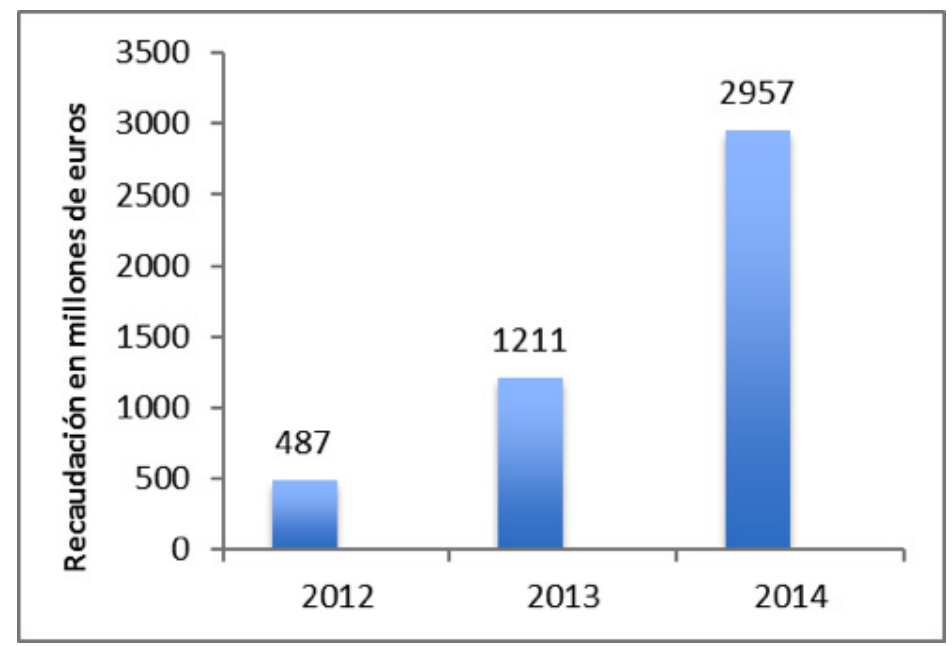

Figura 1: Evolución en la recaudación vía crowdfunding en Europa 20122014. Fuente: elaborado a partir de Wardrop et al. (2015). 
El papel de la comunicación en el crowdfunding... 284

MONOGRÁFICO

El término crowdfunding abarca cuatro modelos de recaudación financiera: recompensas, donaciones, inversiones y préstamos (Barabas, 2012; Beaulieu, Sarker \& Sarker, 2015; Belleflamme, 0mrani \& Peitz, 2015). Estos modelos son tan distintos entre sí que algunos autores los consideran como negocios distintos catalogados bajo un mismo nombre (Hemer, 2011). A continuación se detallan cada uno de ellos y se presenta su importancia cuantitativa a nivel internacional.

1. Recompensas o reward crowdfunding. Es el modelo más utilizado (Belleflamme, Lambert \& Schwienbacher, 2014) con un peso del 43\% sobre la recaudación total de capital (Massolution, 2013). Consiste en la provisión de financiación a un proyecto a cambio de la promesa de entrega de una contraprestación que nunca puede ser de carácter económico. Incluye tres tipos de recompensas para los mecenas: recompensas simples, patrocinios y pre-ventas.

- Las recompensas simples suelen ser artículos materiales que se prometen a cambio de apoyar el proyecto, por ejemplo, una camiseta.

- Los patrocinios ofrecen visibilidad pública a aquellos que contribuyen a la financiación del proyecto (Zheng et al., 2015). Un ejemplo sería la aparición de los nombres de los mecenas en los créditos de la película que han patrocinado.

- La pre-venta consiste en la provisión de financiación para costear la producción del artículo que el proyecto respalda (Hemer, 2011).

2. Donaciones o donation crowdfunding. Consiste en la provisión altruista de financiación a un proyecto y representa el $29 \%$ sobre el total de fondos recaudados a través de crowdfunding. Este modelo es habitual en proyectos culturales y en los de carácter social (Mata, 2014).

3. Inversiones de capital o equity crowdfunding. Se trata de una financiación participativa con un peso del 15\%. Se caracteriza porque el mecenas, inversor para este modelo específicamente, puede adquirir un porcentaje de la 


\section{MONOGRÁFICO}

titularidad sobre los derechos, acciones o participaciones del proyecto o de la empresa promotora (Ley 5/2015, de 27 de abril, de fomento de la financiación empresarial).

4. Préstamos o debt crowdfunding. Este modelo recoge el $13 \%$ de las recaudaciones vía crowdfunding. El mecenas es un prestamista online que acuerda la recuperación de su inversión con una determinada rentabilidad (Gracía Labarta, 2014).

Los modelos debt y equity crowdfunding fomentan operaciones en las que las compensaciones a los contribuyentes son los resultados económicos de las mismas (Gracía Labarta, 2014). Por el contrario, en los modelos reward y donation crowdfunding, las recompensas son fijadas y mostradas a los potenciales mecenas previamente.

Una vez presentados los modelos, es interesante conocer los motivos que llevan a participar a promotores y mecenas en el crowdfunding ya que se trata de una actividad más compleja que la pura recaudación de fondos:

- Los promotores publican los proyectos en la red y buscan uno o varios de los siguientes resultados: reacción previa del público, testeo de ideas, financiación, visibilidad pública y/u obtención de feedback (Willems, 2013; Sahm et al., 2014).

- Los financiadores, aquellos que se comprometen económicamente, lo hacen por el retorno prometido a cambio de su aportación y/o por el sentimiento de pertenencia a una comunidad de individuos con intereses y pasiones compartidas (Hemer, 2011; Willems, 2013; Stengel, 2014).

Habitualmente los promotores utilizan Internet para dar a conocer sus proyectos $\mathrm{y}$, aunque pueden hacerlo de forma independiente creando su propia página web, la mayoría opta por las plataformas de crowdfunding disponibles online para conseguir alcanzar e influir en el mayor número de potenciales mecenas. Desde estas plataformas, los promotores han de exponer sus proyectos mediante una eficiente 
El papel de la comunicación en el crowdfunding... 286

campaña de comunicación persuasiva. Se entiende por comunicación persuasiva "la intención consciente de un individuo que pretende modificar la conducta de otro individuo o grupo a través de un mensaje y con la utilización del lenguaje" (Niño González, García Guardia \& Alcaraz Lladró, 2014: 271). En la actual sociedad de la información, esta comunicación persuasiva se ha de adaptar a los nuevos entornos (plataformas de crowdfunding en este estudio). La posibilidad de establecer un diálogo personalizado en tiempo real con los mecenas induce a modificar las formas que tiene que adoptar la comunicación y la retórica (Berlanga, 2013).

La capacidad de persuasión de un mensaje se puede medir, de acuerdo con Renvoise y Morin (2007), analizando el uso de un conjunto de factores que actúan como incentivos para animar al cerebro primitivo en la toma de decisiones:

- Egocentrismo. El foco de todo mensaje tiene que estar puesto en el potencial cliente. Kruger, et al (2005) definen egocentrismo del emisor como la dificultad de desprenderse del propio punto de vista y centrarse en la perspectiva del receptor. En su trabajo sobre la comunicación por correo electrónico muestran como el egocentrismo del emisor influye negativamente en la recepción del mensaje. Por otra parte, se considera que la nueva audiencia de las redes sociales se basa en el egocentrismo (Flores Vivar, 2009).

- Contraste. El contraste facilita las decisiones. Si el mensaje no es claro, la toma de decisiones se ralentiza. La toma de decisiones innovadoras hace que el cerebro libere dopamina, haciendo que el sujeto se sienta bien (Wittmann et al., 2008).

- Tangibilidad. Consiste en la posibilidad de que algo pueda ser percibido de manera precisa (Real Academia Española, 2015). Existe una relación positiva entre tangibilidad y generosidad (Cryder \& Loewenstein, 2011) por lo que se puede pensar que se deberían priorizar los conceptos tangibles y concretos sobre los abstractos y complejos (Agarwal, 2014; Georges, Bayle-Tourtoulou \& Badoc, 2014).

- Comienzos y finales. Hace referencia a la importancia de la primera impresión y a la tendencia de las personas a recordar las huellas que deja el final de un comunicado y no sus partes intermedias (Renvoise \& Morin, 2007). 
- Estímulos visuales. En la toma de decisiones las imágenes prevalecen sobre las palabras (Renvoise \& Morin, 2007). En este sentido, la investigación realizada por Niño González, García Guardia y Alcaraz Lladró (2014) concluye que los anuncios publicitarios en el móvil que mejor se entienden y más se recuerdan son los que combinan texto e imagen.

- Emoción. La toma de decisiones tiene su base en las emociones y, posteriormente, aparece un intento de justificación racional (Renvoise \& Morin, 2007).

Cialdini (1993, 2001), desde el campo de la psicología, propone otro conjunto de principios que se han denominado "armas de persuasión" debido a que apelan a los instintos como motivadores del comportamiento:

- Agrado. Propensión a estar de acuerdo con aquellas personas hacia las que se siente simpatía, agradan o son similares a uno mismo.

- Aprobación social. Los sujetos tienden a comportamientos en concordancia a un determinado (sub)grupo social para sentirse aceptados y sentir que se reduce el riesgo de tomar decisiones equivocadas. Además se tiende a tener más confianza en mensajes respaldados por grandes grupos, expertos o celebridades.

- Autoridad. Los sujetos tienden a confiar en emisores con credibilidad y experiencia. Este principio se cumple especialmente cuando el receptor no es un experto. Para ilustrar este principio se pueden utilizar frases como “dicen los científicos" o "una reciente investigación muestra...". La ropa, la posición o los accesorios pueden ser símbolos de autoridad. Pocos temas han recibido tanta atención por parte de los investigadores de la comunicación como "ethos", o más comúnmente "credibilidad de la fuente", cuyo origen está en la visión de Aristóteles acerca de que el "ethos" es el medio más potente de la persuasión (ej. Vitale, 2013; Reinares-Lara, Martín-Santana \& Muela-Molina, 2016). 
El papel de la comunicación en el crowdfunding... 288

MONOGRÁFICO

- Exclusividad y escasez. Hacer sentir especial al potencial cliente ofreciendo productos/servicios únicos o en unidades limitadas. Las ofertas exclusivas y difíciles de conseguir resultan más atractivas ya que se tiende a suponer que son mejores que las que están fácilmente disponibles.

- Reciprocidad. Los sujetos tienden a pagar lo que reciben de los demás. Cuando el emisor ofrece algo en primer lugar, los receptores van a sentir una sensación de endeudamiento, que hará que sean más propensos a cumplir con las peticiones posteriores del emisor.

- Consistencia. Existe una tendencia a comportarse de acuerdo a compromisos previos. Las audiencias reciben cientos de opciones que implican nuevas decisiones pero por conveniencia, se toma una sola decisión y luego se adhieren a ella para decisiones posteriores. El compromiso puede tener un efecto poderoso en el comportamiento futuro.

Estos principios son poderosos porque evitan la parte racional del cerebro apelando a los instintos (Cialdini 2011) aunque para que funcionen es necesario que el oferente se apoye en un producto que aporte valor real a los clientes.

\section{Material y métodos}

Este estudio parte de la hipótesis de que el mensaje de una campaña de comunicación de crowdfunding afecta al éxito de la recaudación de fondos. En este sentido, el objetivo general del presente trabajo es analizar la influencia de la comunicación en la captación de fondos.

Este objetivo general se concreta en los siguientes objetivos específicos:

- Analizar cómo las campañas de comunicación de crowfunding utilizan los factores de la persuasión. 
- Realizar un análisis comparativo de la utilización de dichos factores en dos campañas con distinto resultados.

- Ofrecer recomendaciones para la elaboración de una estrategia de comunicación exitosa para campañas de crowdfunding basadas en recompensas.

Para dar respuesta a estos objetivos se ha optado por un enfoque cualitativo utilizando la metodología del caso. Esta metodología se concibe como una estrategia práctica investigadora dirigida a comprender las dinámicas actuales en contextos específicos (Carazo, 2006).

Para la elección del caso, se consideraron dos requisitos:

- Haber sido publicado en alguna plataforma de crowdfunding de recompensas ya que el poder recaudatorio de este modelo representa el $43 \%$ a nivel global (Massolution, 2013).

- Debía corresponderse con un proyecto lanzado más de una vez por sus promotores buscando mejorar y/o conseguir la financiación. Para que esto sucediera, la primera campaña debía haber fallado en su intento de financiación y haber conseguido el éxito en una campaña posterior. De esta forma, se podría realizar el análisis comparativo de las campañas de comunicación.

Se seleccionó el caso Coolest que se lanzó en la plataforma virtual Kickstarter. Coolest es una nevera portátil con una licuadora, un altavoz extraíble y resistente al agua que funciona con Bluetooth, un cargador USB, ruedas especiales para facilitar su transporte, un abridor de botellas, entre sus principales atributos. Este proyecto se lanzó en dos ocasiones: la primera campaña no alcanzó el éxito (en adelante campaña 1) al no conseguir el mínimo de financiación fijado; la campaña posterior (en adelante campaña 2) alcanzó la suma de 13.285.226 dólares americanos, batiendo, en su momento, el récord histórico de captación de fondos a través de crowdfunding (Tabla 1). 


\begin{tabular}{|c|c|c|}
\hline $\begin{array}{l}\text { INFORMACIÓN DE LAS } \\
\text { CAMPAÑAS } \\
\text { COOLEST }\end{array}$ & $=$ & Codent \\
\hline & CAMPAÑA 1 & CAMPAÑA 2 \\
\hline Nombre & $\begin{array}{l}\text { The Coolest: Cooler with Blen- } \\
\text { der, Music and So Much More. }\end{array}$ & $\begin{array}{c}\text { COOLEST COOLER: } 21 \mathrm{~s} \\
\text { Century Cooler that's Actually } \\
\text { Cooler. }\end{array}$ \\
\hline Periodo de financiación & $\begin{array}{l}\text { 26/11/2013 - 26/12/2013 } \\
31 \text { días en invierno. }\end{array}$ & $\begin{array}{c}8 / 07 / 2014 \text { - 30/08/2014 } \\
52 \text { días en verano. }\end{array}$ \\
\hline Objetivo de financiación & 125.000 dólares & 50.000 dólares \\
\hline $\begin{array}{l}\text { Dinero comprometido y por- } \\
\text { centaje sobre el objetivo de } \\
\text { financiación. } \\
\text { Regla del todo o nada }\end{array}$ & $\begin{array}{c}102.188 \text { dólares } \\
81,75 \%<100 \% \\
\text { Resultado: no financiado }\end{array}$ & $\begin{array}{c}\text { 13.285.226 dólares } \\
26.570 \%>100 \% \\
\text { Resultado: } 13.285 .226 \text { dólares }\end{array}$ \\
\hline Fuente de información & https://goo.gl/1nbQ3p & https://goo.gl/v0MCZz \\
\hline
\end{tabular}

Tabla 1: Ficha técnica y unidades de análisis. Fuente: elaboración propia a partir de la plataforma de Kickstarter.

En la plataforma Kickstarter, el promonotor publica de cada proyecto un vídeo promocional y un texto explicativo. Los vídeos cuentan con tres elementos fundamentales: sucesos, personajes y escenarios (Fernández-Cañaveral, 2010). El texto se ilustra con elementos visuales. Estos elementos de comunicación serán la base del estudio de las dos campañas y su contenido será evaluado a la luz de los factores de persuasión.

\section{Resultados}

En las siguientes líneas se presentan los resultados del análisis comparativo de los dos videos y de los dos textos correspondientes a las dos campañas tomando 
como referencia los factores determinantes de la capacidad de persuasión descritos por Cialdini (1993, 2001), Renvoise y Morin (2007), Wittmann et al., (2008), Cryder y Loewenstein (2011), Georges, Bayle-Tourtoulou y Badoc, (2014).

Egocentrismo. La comunicación de la campaña 1 se realiza en primera persona y se centra en los creadores del proyecto. La campaña 2 es un proyecto creado para el público mecenas y por ello utiliza la segunda persona y lo implica de forma directa y continua (Tabla 2). Además, la campaña 2 potencia el egocentrismo de los mecenas realizando una pronta presentación descriptiva y figurativa de las recompensas para atraer su atención. Sin embargo, en la campaña 1 las recompensas aparecen al final, son menos llamativas y prevalece la información descriptiva sobre el producto y el proyecto.

\begin{tabular}{|c|c|}
\hline Campaña 1 & Campaña 2 \\
\hline $\begin{array}{c}\text { "This is where you come in. If we can reach our } \\
\text { investment goal of } \$ 125,000 \text {, then we can bring } \\
\text { The Coolest (...)" }\end{array}$ & $\begin{array}{c}\text { "We want you to have your COOLEST as soon as } \\
\text { possible." }\end{array}$ \\
\hline $\begin{array}{c}\text { "(...) can find a reward level that helps us meet } \\
\text { our goal..." }\end{array}$ & $\begin{array}{c}\text { "(...) for just a hundred and eighty dollars you } \\
\text { can be one of the first people in the world with a } \\
\text { cooler that's actually cool." }\end{array}$ \\
\hline $\begin{array}{l}\text { "Luckily, I have experience fast-tracking other } \\
\text { products to market. Our CAD models are already } \\
\text { designed, and we have sourcing redundancies } \\
\text { for each major component. We are working } \\
\text { with a leading manufacturing design firm and } \\
\text { have developed a strategic partnership with the } \\
\text { category leader in commercial manufacturing to } \\
\text { make the 'Coolest'... well, the coolest! [...]We } \\
\text { are close but we still need your help to: [...]" }\end{array}$ & $\begin{array}{l}\text { "Normal coolers are boring break easily and are } \\
\text { a pain to get to and from your destination. [...] } \\
\text { You got this } 18 \mathrm{~V} \text { rechargeable blender. [...] You } \\
\text { are already carrying around a cooler full of ice } \\
\text { and tasty beverages, why don't blend them up } \\
\text { and become summer-time hero anytime, anywhe- } \\
\text { re? [...] And since you have this } 18 \mathrm{~V} \text { battery for } \\
\text { the blender, why not to make the most out of it? } \\
\text { Maybe your camera battery is low, or maybe you } \\
\text { have and iPhone and wanna use it after two in } \\
\text { the afternoon. Recharge your gear wherever you } \\
\text { are with this waterproof USB charger." }\end{array}$ \\
\hline $\begin{array}{l}\text { Las recompensas para los mecenas figuran al } \\
\text { final del texto. }\end{array}$ & $\begin{array}{l}\text { Tras una descripción breve y general del produc- } \\
\text { to se presentan detalladamente las recompensas. }\end{array}$ \\
\hline
\end{tabular}

Tabla 2: Muestras de egocentrismo. Cambio del foco del mensaje: de los creadores del proyecto ("we") a los potenciales mecenas ("you"). Fuente: elaboración propia a partir de la transcripción del audio del video de la plataforma de Kickstarter. 
El papel de la comunicación en el crowdfunding... $\mid 292$

MONOGRÁFICO

Contraste. Alude a la exclusividad del producto como elemento de persuasión (Cialdini, 2001; Wittmann et al., 2008). En ambas campañas se habla de los defectos de las neveras tradicionales y en la campaña 2 se presenta la novedad de forma más atractiva (principio de agrado y aprobación social de Cialdini): “Las neveras tradicionales son aburridas, se rompen fácilmente y para llevar hielo son incómodas. La nueva nevera contiene 60 litros impresionantes, llenos de tanta diversión que usted buscará excusas para salir de casa con más frecuencia". A continuación, se dirige a los mecenas con ideas nuevas sobre lo que podrían conseguir con la nueva nevera.

Tangibilidad. Se ha evaluado sobre el plan de ejecución del proyecto establecido para cada campaña: la primera contiene mensajes contradictorios sobre el plan de ejecución del proyecto ("Estamos cerca, pero todavía necesitamos su ayuda para... "). La campaña 2, al mostrar un plan listo y completo, aumenta la confianza del público (“Como se puede ver, tenemos nuestro diseño finalizado y listo para la gran liga... queremos que usted tenga su COOLEST tan pronto como sea posible"). Además, habla de que las recompensas se entregarán a tiempo y con la máxima calidad.

Comienzos y finales. Para comunicar es necesario, en primer lugar, captar la atención, después mantenerla y concluir con una llamada a la acción. Mientras que la campaña 1 presenta el nuevo producto con un título descriptivo ("The Coolest: Cooler with Blender, Music and So Much More"), la campaña 2, utiliza un título emotivo y capta la atención del lector con el uso de mayúsculas y un juego de palabras ("COOLEST COOLER: 21st Century Cooler that's Actually Cooler"). Como se ha comentado, la segunda campaña presenta la nueva nevera como "60 litros impresionantes, llenos de tanta diversión que usted buscará excusas para salir..." en un entorno más lúdico y luminoso (verano). Este comienzo llama mucho más la atención de los mecenas espectadores que el de la primera.

El vídeo de la segunda campaña finaliza con una llamada a la acción: “Comparte este vídeo ahora para que podamos realizar tu nevera" y fue compartido 389.547 veces en Facebook. Sin embargo, en la primera campaña, el vídeo finaliza con una pantalla en negro y se compartió 831 veces en Facebook.

Para mantener la atención de los mecenas, la estructura del vídeo y del texto en la plataforma es fundamental (Tabla 3).

DOI: ri14.v14i2.968 | ISSN: 1697-8293 | Año 2016 Volumen 14 N² 2 | ICONO14 
MONOGRÁFICO

\begin{tabular}{|c|c|}
\hline Campaña 1 & Campaña 2 \\
\hline $\begin{array}{l}\text { The Coolest: Cooler with Blender, Music and So } \\
\text { Much More } \\
\text { (10 palabras, } 56 \text { caracteres) }\end{array}$ & $\begin{array}{c}\text { COOLEST COOLER: 21st Century Cooler that's } \\
\text { Actually Cooler } \\
\text { (8 palabras, } 58 \text { caracteres) }\end{array}$ \\
\hline $\begin{array}{l}\text { 1. Introducción impersonal. } \\
\text { 2. Comentarios de cinco empresas sobre las } \\
\text { bondades de la nueva propuesta de pro- } \\
\text { ducto. Prevalece el texto sobre la imagen. } \\
\text { 3. Descripción textual detallada de los atribu- } \\
\text { tos de la nevera. } \\
\text { 4. Plan de ejecución del proyecto. } \\
\text { 5. Recompensas ofrecidas a los mecenas. } \\
\text { 6. Lista de los atributos del producto. } \\
\text { 7. Riesgos y retos. } \\
\text { 8. Sin lista de preguntas frecuentes. }\end{array}$ & $\begin{array}{l}\text { 1. Introducción dirigida a los mecenas. } \\
\text { 2. Comentarios de cuarenta empresas intro- } \\
\text { ducidos con la siguiente frase: "Mira quién } \\
\text { está hablando sobre Coolest". Muestra los } \\
\text { logotipos de empresas con gran notoriedad. } \\
\text { Prevalece la imagen sobre el texto. } \\
\text { 3. Lista de iconos que permiten visualizar y } \\
\text { entender rápidamente los atributos novedo- } \\
\text { sos de la nevera. } \\
\text { 4. Recompensas ofrecidas a los mecenas. } \\
\text { 5. Descripción detallada de los atributos del } \\
\text { 6. Producto con fotografías. } \\
\text { 7. Riesgos y retos. } \\
\text { 8. Lista de } 25 \text { preguntas frecuentes. }\end{array}$ \\
\hline
\end{tabular}

Tabla 3: Estructura del texto de las campañas en la plataforma de Kickstarter. Fuente: elaboración propia.

Se observa un cambio en la organización de la información que facilita y agiliza la comprensión del proyecto. Efectivamente, también en este campo se confirma que "la potencialidad de la imagen, la palabra, el relato... no serían nada sin una narración que vertebre todos los elementos y se enriquezca con recursos expresivos procedentes de los territorios del lenguaje visual, icónico, simbólico, gestual y por supuesto, verbal" (Fernández Fernández, Baños González \& García García, 2014: 400).

Estímulos visuales. En la segunda campaña se mejora la producción del vídeo y la fotografía. Efectivamente, las escenas del vídeo son más luminosas y las imágenes de las características del producto y de las recompensas son más atractivas (Tabla 4). Por todo lo anterior, se puede concluir que la campaña 2 es más visual (17 fotografías en la web) que la inicial (13 fotografias). En línea con lo que sucede en publicidad y en otros campos de comunicación, también se observa cómo las nuevas tecnologías favorecen el uso de la manipulación de la imagen con vistas a seducir la mirada del espectador resaltando elementos atractivos (Arroyo, 2005; Gómez Martínez, 2013). 


\begin{tabular}{|c|c|c|c|}
\hline \multicolumn{2}{|c|}{ Campaña 1} & \multicolumn{2}{|c|}{ Campaña 2} \\
\hline \multicolumn{4}{|c|}{ Videos de las campañas } \\
\hline \multicolumn{2}{|c|}{$\begin{array}{l}\text { El vídeo se ambienta en un parque y en un día } \\
\text { frío. El creador del proyecto presenta los atribu- } \\
\text { tos del producto. }\end{array}$} & \multicolumn{2}{|c|}{$\begin{array}{l}\text { El vídeo muestra un día luminoso de verano y } \\
\text { asocia distintos ambientes con cada una de las } \\
\text { utilidades de la nueva nevera. El vídeo revela } \\
\text { cómo los usuarios disfrutan de los atributos de } \\
\text { la nevera mientras una voz en off los describe. }\end{array}$} \\
\hline \multicolumn{4}{|c|}{ Texto de las campañas } \\
\hline \multicolumn{2}{|c|}{$\begin{array}{l}\text { El listado de atributos del producto se presenta al } \\
\text { final del texto de ventas de la web en texto plano: } \\
\text { - } 18 \text { volt battery powered, full size blender, } \\
\text { capable of } 60+\text { batches per charge. } \\
\text { - Rechargeable, removable Bluetooth music } \\
\text { player capable of operating for over } 8 \text { hours } \\
\text { per charge, while streaming music from up to } \\
50 \text { feet away. High strength, locking bungee } \\
\text { tie-down. } \\
\text { - Virtually unbreakable LED lighting recessed } \\
\text { and waterproof in the lid. Built in bottle-top } \\
\text { opener. } \\
\text { - Fully customizable color skins, available in } \\
36 \text { colors choices. } \\
60 \text { quart, hard sided, rolling cooler (20\% } \\
\text { larger than my prototype). } \\
\text { Reinforced, easy rolling wheels" }\end{array}$} & $\begin{array}{l}\text { El listado de atributos } \\
\text { del producto, en formato } \\
\text { imagen, se incluye al co- } \\
\text { mienzo de la descripción } \\
\text { del producto para ofrecer } \\
\text { una visión de conjunto al } \\
\text { lector antes de introducir } \\
\text { las descripciones detalla- } \\
\text { das de cada uno de ellos }\end{array}$ & 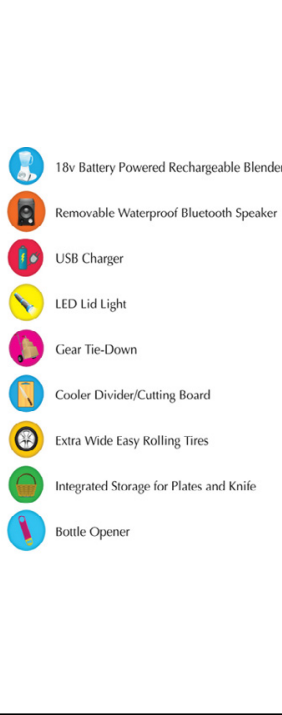 \\
\hline $\begin{array}{l}\text { Las recompensas prometidas } \\
\text { para los mecenas se presen- } \\
\text { tan dibujadas e incluyen } \\
\text { largos textos descriptivos. } \\
\text { El tamaño de la tipografía } \\
\text { de los números que indican } \\
\text { la cantidad de dinero a } \\
\text { comprometer es pequeño. } \\
\text { Los colores de fondo utilizados } \\
\text { dificultan la lectura del texto. }\end{array}$ & 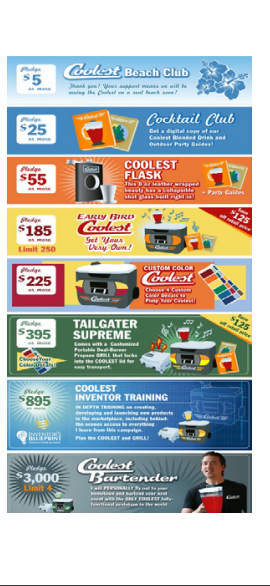 & $\begin{array}{l}\text { Las recompensas prometidas } \\
\text { se presentan con fotografías } \\
\text { que muestran la apariencia } \\
\text { real de las recompensas e } \\
\text { intentan aumentar el interés } \\
\text { de los mecenas. Los textos } \\
\text { que las acompañan son } \\
\text { breves y concisos. } \\
\text { El tamaño de la tipografía } \\
\text { de los números que indican } \\
\text { la cantidad de dinero a } \\
\text { comprometer es grande. } \\
\text { Un fondo blanco facilita la } \\
\text { lectura del texto. }\end{array}$ & 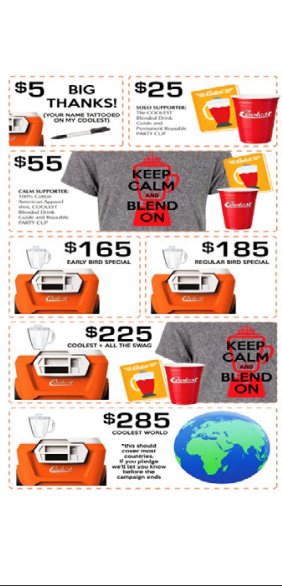 \\
\hline
\end{tabular}

Tabla 4: Análisis comparativo de los estímulos visuales.

Fuente: elaboración propia a partir de los vídeos y de los textos explicativos de las campañas publicados en la plataforma de Kickstarter. 
Emoción. Mientras que la campaña 1 describe el producto y enumera sus atributos físicos, la campaña 2 recuerda a los mecenas los inconvenientes de las neveras tradicionales para, después, presentar las soluciones que aporta la nueva nevera (Tabla 5).

\begin{tabular}{|c|c|}
\hline Campaña 1 & Campaña 2 \\
\hline $\begin{array}{l}\text { "This insulated box has been called cooler for } \\
\text { over sixty years. But what's really cooler then? } \\
\text { [...] The Coolest is a complete refresh of what } \\
\text { a portable cooler can be. The first big improve- } \\
\text { ment is the } 18 \text { volts battery powered blender. } \\
\text { [...] And did you know that no percent of coo- } \\
\text { lers come with a Bluetooth speaker built right } \\
\text { in? Well, The Coolest changes all that. [...] I } \\
\text { wanted to make sure that The Coolest elimina- } \\
\text { ted all those annoying shortfalls that I have } \\
\text { noticed with other coolers over the years." } \\
\text { annoying shortfalls that I have noticed with } \\
\text { other coolers over the years." }\end{array}$ & $\begin{array}{l}\text { "Regular coolers are boring, break easily and are } \\
\text { a hassle to haul around just to carry the ice. The } \\
\text { COOLEST cooler is } 60 \text { quarts of AWESOME packed } \\
\text { with so much fun you'll look for excuses to get } \\
\text { outside more often. Maybe you want to use the } \\
\text { built-in ice crushing blender to whip up some } \\
\text { margaritas or smoothies on your next boat trip } \\
\text { or tailgate? Maybe you just want to always have } \\
\text { music on hand with the waterproof Bluetooth } \\
\text { speaker or want to recharge your electronics with } \\
\text { the built in USB charger?" } \\
\text { the waterproof Bluetooth speaker or want to } \\
\text { recharge your electronics with the built in USB } \\
\text { charger?" }\end{array}$ \\
\hline
\end{tabular}

Tabla 5: Muestras de la utilización del contraste y las emociones en la comunicación de las campañas en la plataforma de Kickstarter. Fuente: elaboración propia a partir de la transcripción del audio del video.

Se introducen cambios en los mensajes verbales, no verbales y paraverbales que transmiten emociones más positivas. El entorno del segundo vídeo trabaja el sentido de pertenencia a un grupo y busca la creación de un vínculo emocional con el potencial contribuyente.

Respecto a los principios de persuasión propuestos por Cialdini (2001) se observa que en la segunda campaña se presentan todos ellos con mayor intensidad: el agrado aparece cuando se muestran imágenes más emotivas y evocadoras de sensaciones positivas; la aprobación social se refleja en el vídeo con grupos de personas felices que disfrutan alrededor de la nueva nevera; la autoridad se manifiesta cuando el promotor cuenta experiencias previas de éxito y da una información más completa de las empresas de reconocido prestigio que apoyan y colaboran en el proyecto (Tabla 6); la exclusividad y escasez se muestra cuando el promotor brinda a los mecenas la posibilidad de ser los primeros en disponer de la novedosa nevera; la reprocidad se muestra en el 
vídeo destacando las recompensas desde el inicio; la consistencia, invitando a compartir el vídeo.

\begin{tabular}{|c|c|}
\hline Campaña 1 & Campaña 2 \\
\hline 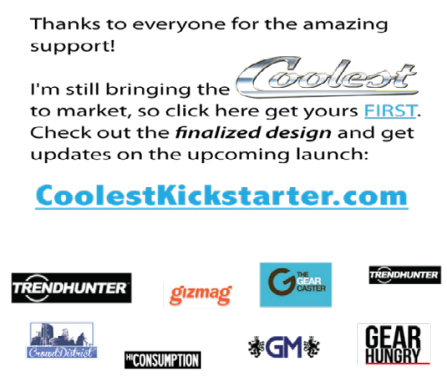 & 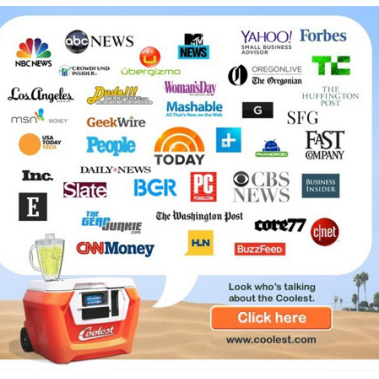 \\
\hline
\end{tabular}

Tabla 6: Muestras de la utilización del principio de autoridad en el texto de las campañas en la plataforma de Kickstarter. Fuente: elaboración propia.

\section{Discusión}

La importancia del crowdfunding como medio de financiación ha crecido en los últimos años y todo apunta a que lo seguirá haciendo en los años venideros debido al creciente protagonismo de Internet, de las plataformas y de las redes sociales (Lee, DeWester \& Park, 2008; Wardrop et al., 2015). El crowdfunding se está consolidando como uno de los caminos para costear el emprendimiento (Kleemann, Voss \& Rieder, 2008; Schwienbacher \& Larralde, 2010). En esta forma colectiva, participativa y colaborativa de recaudar fondos, el público se convierte en mecenas de un proyecto si su creador logra convencer. En consecuencia, además de contar con un buen proyecto, es muy importante comunicarlo de forma adecuada. La comunicación representa una pieza clave para alcanzar un objetivo específico y recaudar la financiación necesaria. $\mathrm{Si}$ bien los cambios tecnológicos se han constituido como importantes motores del desarrollo de las plataformas de crowdfunding, el contenido del mensaje sigue siendo un elemento troncal que dota de significado a la comunicación.

El estudio desvela interesantes recomendaciones en la aplicación de los factores de la persuasión de Cialdini (2001) y Renvoise y Morin (2007) en un área relativamente novedosa como es el crowdfunding: 
- En el mensaje se debe cuidar el comienzo y el final. Las primeras palabras captarán la atención y con el final se dejará la huella a seguir. Se trata de atraer a los mecenas desde el comienzo. Crear una primera impresión buena es determinante para el éxito de una campaña de crowdfunding. Elementos como el título o la introducción del proyecto influyen sobre el mecenas espectador para que siga leyendo o haga "clic" para regresar al menú principal de la plataforma y abandonar. Un título corto y emotivo junto a estímulos visuales atractivos han funcionado. En el cierre, hay que destacar la tendencia de las personas a recordar las huellas que deja el final (Renvoise \& Morin, 2007) y se ha visto cómo invitar al espectador a compartir el proyecto y participar produce efectos en las redes sociales.

- Tras la primera conexión con los mecenas, es necesario mantener la atención hasta la llamada final a la acción. Se recomienda cuidar la estructura del mensaje utilizando las herramientas de la comunicación persuasiva para organizar su contenido.

- El foco del mensaje tiene que estar puesto en el mecenas (egocentrismo): los interesantes beneficios que aporta el producto deben dirigirse al mecenas: "tú puedes conseguirlos" en vez de "el producto tiene y/o puede".

- Es esencial que se describan los beneficios y las soluciones que los mecenas pueden encontrar con el nuevo producto en contraste con los problemas que pueden estar teniendo hasta el momento. El mensaje debe poner su acento, más que en una lista de prestaciones y características, en las soluciones innovadoras que derivan de dichas características (Cámara \& Sanz, 2001). Estos resultados concuerdan con la tesis que indica que cuando un sujeto toma decisiones innovadoras (comprar la nueva nevera) el cerebro libera dopamina, haciendo que se sienta bien (Wittmann et al., 2008).

- La tangibilidad, la concreción de un proyecto listo para su puesta en marcha, es otro elemento esencial para potenciar la confianza de los mecenas. Los resultados de las campañas analizadas apoyan una relación positiva entre tangibilidad y generosidad señalada por autores como Cryder y Loewenstein (2011) o Georges, Bayle-Tourtoulou y Badoc (2014). En consecuencia, se recomienda 
El papel de la comunicación en el crowdfunding... 298

trabajar el mensaje con elementos concretos y completos (por ejemplo, plan de ejecución completo o soluciones para situaciones concretas).

- Se ha de demostrar autoridad para reforzar la confianza de los mecenas en el proyecto. Esa autoridad ha de proceder de bases firmes como, en este caso, experiencias previas de éxito o contar con colaboradores relevantes en el mercado.

Para cerrar estas conclusiones hay que destacar que generar una mezcla de atracción, confianza y emoción ha contribuido al éxito de la segunda campaña.

\subsection{Limitaciones y futuras líneas de investigación}

Este estudio presenta una serie de limitaciones que abren futuras líneas de investigación. Por un lado, se han considerado los productos presentados en las campañas uno y dos como idénticos aunque, como se puede apreciar en la ficha técnica, existen algunas modificaciones en el diseño. Futuros trabajos podrían analizar el efecto del diseño en la aceptación de los proyectos. Por otro lado, para la elaboración de este estudio no se ha realizado un análisis off-page. Toda la información ha sido tomada de las páginas web de cada una de las campañas en Kickstarter. Sin embargo, parte de la comunicación promocional llevada a cabo por el equipo creador del proyecto se hizo fuera de la plataforma Kickstarter, lo que directamente lleva a proponer un estudio sobre el efecto, alcance e importancia de las variables off-the-page en las campañas de crowdfunding. Puede ser de especial interés el análisis del rol y cobertura de las redes sociales.

La literatura muestra los motivos que llevan a los promotores y mecenas a participar en los proyectos (Willems, 2013; Belleflamme, Lambert \& Schwienbacher, 2014; Stengel, 2014). Una línea futura podría analizar el papel particular de los motivos en un caso como éste ya que los perfiles de los mecenas pueden ser diversos y existen pocos estudios que permitan explicar cuáles son sus principales motivaciones (Braet, Spek \& Pauwels, 2013). Otra línea futura puede ser comparar estos resultados con los obtenidos de aplicar las nuevas herramientas de neuromarketing (Gómez \& Bandrés, 2014).

Por último, la literatura distingue entre los mensajes verbales (aquellos configurados por el contenido y la forma), mensajes no verbales (aquellos transmitidos

DOI: ri14.v14i2.968 | ISSN: 1697-8293 | Año 2016 Volumen 14 NN$^{\circ} 2$ | ICONO14 


\section{MONOGRAFICO}

a través del lenguaje corporal, que incluye expresión facial, posturas y gestos) y mensajes paraverbales (se transmiten a través del timbre, tono de la voz y la velocidad de palabra) (MVC, 2013; Windle \& Warren, 2014). Sería muy interesante analizar la comunicación no verbal y paraverbal de los promotores.

\section{Anexos}

[1] https://figshare.com/s/a0a86fb0e296d8440ad6

\section{Notas}

[1] La Asociación Española de Crowdfunding (2013) ha propuesto denominar mecenas a los individuos que hacen aportaciones.

\section{Referencias}

Agarwal, S. (2014). Neuromarketing in Action: How to Talk and Sell to the Brain. Journal of Consumer Marketing, 31(5), 404-405. doi: ttp://dx.doi.org/10.1108/ JCM-02-2014-0879

Arroyo Almaraz, I. (2005). Creatividad publicitaria y retórica. De la metáfora a los efectos especiales. Icono 14, 3 (1), 155-170.

Asociación Española de Crowdfunding. (2013). Información básica sobre el crowdfunding. Asociación Española de Crowdfunding. Recuperado de http://goo.gl/vX1W40 Asociación Española de Crowdfunding. (2014). Informe de la encuesta de la Asociación Española de Crowdfunding sobre las plataformas españolas de Crowdfunding. Recuperado de http://goo.gl/DbRxHU

Barabas, R. L. (2012). Crowdfunding: Trends and Developments Impacting Entertainment Entrepreneurs. NYSBA Entertainment, Arts and Sports Law Journal, 23(2), 38-40. Recuperado de http://goo.gl/66j0H5

Beaulieu, T. Y., Sarker, S., \& Sarker, S. (2015). A conceptual framework for understanding crowdfunding. Communications of the Association for Information Systems, 37, 1-31. Belleflamme, P., Lambert, T., \& Schwienbacher, A. (2014). Crowdfunding: Tapping the right crowd. Journal of Business Venturing, 29(5), 585-609. doi:10.1016/j. jbusvent.2013.07.003 
El papel de la comunicación en el crowdfunding... 300

Belleflamme, P., Omrani, N., \& Peitz, M. (2015). The economics of crowdfunding platforms. Information Economics and Policy, 33, 11-28. doi:10.1016/j.infoecopol.2015.08.003 Berlanga, I. (2013). Retórica clásica y redes on line: dos realidades convergentes y análogas. Perspectivas y prospectivas de 9 expertos en Comunicación. Icono 14, 11 (1), 45-70. doi: 10.7195/ri14.v11i1.548

Braet, 0., Spek, S., \& Pauwels, C. (2013). Crowdfunding the movies: A business analysis of crowdfinanced moviemaking in small geographical markets. Journal of Media Business Studies, 10(1), 1-23.

Cámara, D., \& Sanz, M. (2001). Dirección de ventas. Vender y fidelizar en el nuevo milenio. Prentice Hall. Madrid.

Carazo, P. C. (2006). El método de estudio de caso Estrategia metodológica de la investigación científica. Revista Científica Pensamiento y Gestión. 20 (1), 165-193. Cetti, M., \& Corso, M., (2014). Crowdfunding - a tool to reframe trust and loyalty. Master Thesis, Copenhagen Business School. Recuperado de http://goo.gl/LYzXbh

Cialdini, R. B. (1993). Influence: The psychology of persuasion. New York: Morrow. Cialdini, R. B. (2001) Harnessing the Science of Persuasion. Harvard Business Review, 79(9), 72-81.

Crowdfundinsider (2015). Massolution Posts Research Findings: Crowdfunding Market Grows 167\% in 2014, Crowdfunding Platforms Raise $\$ 16.2$ Billion. Crowdfundinsider. Recuperado de http://goo.gl/LG797A

Cryder, C., \& Loewenstein, G. (2011). The Critical Link Between Tangibility and Generosity. En D. M. Oppenheimer, \& C. Y. Olivola (Eds.). The science of giving: Experimental approaches to the study of charity (237-251). New York: Taylor and Francis.

Coronado Ruiz, C. C., \& Larrañaga Rubio, J. L. (2015). La financiación por medio del crowdfunding del audiovisual: el caso del cortometraje: Juan y la nube". Documentación de las Ciencias de la Información, 38, 203-221.

Dushnitsky, G., Guerini, M., Piva, E., \& Rossi-Lamastra, C. (2016). Crowd funding in europe: Determinants of platform creation across countries. California Management Review, 58(2), 44-71. doi:10.1525/cmr.2016.58.2.44

Fernández-Cañaveral, D. (2010). El personaje público y los arquetipos: un estudio narrativo de los líderes políticos en las elecciones europeas de 2009 en España. Icono14, 8(2), 38-57. 


\section{MONOGRÁFICO}

Fernández Fernández, P., Baños González, M., \& García García, F. (2014): Análisis iconográfico de la publicidad audiovisual de perfumes. El caso J'Adore. Icono 14, 12(1), 398-430. doi:10.7195/ri14.v12i1.549

Flores Vivar, J. M. (2009). Nuevos modelos de comunicación, perfiles y tendencias en las redes sociales. Comunicar, 33 (17), 73-8. doi:10.3916/c33-2009-02-07

Georges, P. M., Bayle-Tourtoulou, A. S., \& Badoc, M. (2014). Neuromarketing in Action: How to Talk and Sell to the Brain. London: Kogan Page Publishers.

Gómez Martínez, P. J. (2013): Retórica de la historia en la serie "Pulseras Rojas". Un desafío al exceso de las nuevas tecnologías. Icono 14, 11 (1), 125-138. doi: 10.7195/ri14.v11i1.513

Gómez Patiño, M., \& Bandrés Goldáraz, E. (2014): El neuromarketing: Una nueva disciplina para la investigación de audiencias y de la opinión pública. Icono 14, volumen (12), 395-415. doi: 10.7195/ri14.v12i2.666

Google Trends (2015). Google Trends. Recuperado de http://goo.gl/Miv7FP

Gracía Labarta, C. (2014). Presente y futuro del crowdfunding como fuente de financiación de proyectos empresariales. Revista Española de Capital Riesgo, 1, 3-19.

Hemer, J. (2011). A snapshot on crowdfunding. Working Papers Firms and Region. $\mathrm{R} 2 / 2011,1-34$.

Kazmark, J. (2013). Kickstarter before Kickstarter. Recuperado de http://goo. $\mathrm{gl} / 9 \mathrm{cQoFg}$.

Kleemann, Frank, G. Gunter Voss \& Kerstin Rieder. (2008). Un(der)paid Innovators: The Commercial Utilization of Consumer Work through Crowdsourcing. Science, Technology \& Innovation Studies, 4(1), 5-26.

Kruger, J., Epley, N., Parker, J., \& Ng, Z-W. (2005). Egocentrism over E-mail: Can we communicate as well as we think? Journal of Personality and Social Psychology, 89(6), 925-936. doi:10.1037/0022-3514.89.6.925

Lee, S., DeWester, D., \& Park, S. R. (2008). Web 2.0 and opportunities for small businesses. Service Business, 2(4), 335-345. doi: 10.1007/s11628-008-0043-5

Ley 5/2015, de 27 de abril, de fomento de la financiación empresarial. Boletín Oficial del Estado, 28 de abril de 2015, num. 46, pp. 36637-36638. Recuperado de https://goo.gl/RPPT6w

Llorca, G. (2010). El Crowdfunding y los nuevos modelos de negocio info-digital. I Congreso Internacional de Comunicación Audiovisual y Publicidad. Internet y la información, 79-98. Recuperado de http://goo.gl/bZbhu1 
El papel de la comunicación en el crowdfunding... 302

MONOGRÁFICO

Martínez Gallardo, F. D., \& Alberich Pascual, J. (2013). Plataformas y proyectos de crowdsourcing y crowdfunding cinematográfico en España (2007- 2011). Historia y Comunicación Social, 18, 85-95. doi:10.5209/rev_HICS.2013. v18.43950

Martín-Núñez, M. (2014). Pitching 2.0: Conceptualization and develpment in crowdfunding projects. Historia y Comunicacion Social, 19 (1), 821-832. doi: 10.5209/rev-HICS.2014.v19.45005

Massolution (2013). 2013CF. The Crowdfunding Industry Report. Massolution. Recuperado de http://goo.gl/2vm1f3

Mata, J. (2014). Las campañas de crowdfunding: su eficacia en proyectos lucrativos

y causas sociales. Tesis doctoral, Facultat de Comunicació i Relacions Internacionals Blanquerna, Universitat Ramon Llull (Bacelona).

MVC (2013). Communication Strategies. Training Manual, Moreno Valley College. Niño González, J.I., García Guardia, M.L. \& Alcaraz Lladró, A. (2014): Formatos con texto, imagen y sonido en campañas de comunicación persuasiva móvil. Icono 14, 12 (1), 267-292. doi: 10.7195/ri14.v12i1.631

Real Academia Española (2015). Definición de Tangible. Recuperado de http:// lema.rae.es/drae/?val=tangible.

Reinares-Lara, E., Martín-Santana, J. D., \& Muela-Molina, C. (2016). The Effects of Accent, Differentiation, and Stigmatization on Spokesperson Credibility in Radio Advertising. Journal of Global Marketing, 29(1), 15-28.

Renvoise, P., \& Morin, C. (2007). Neuromarketing. Nashville: Thomas Nelson.

Roig Telo, A., Sánchez-Navarro, J., \& Leibovitz, T. (2012). ¡Esta película la hacemos entre todos! Crowdsourcing y crowdfunding como prácticas colaborativas en la producción audiovisual contemporánea. Icono 14, 10(1), 25-40. doi: http:// dx.doi.org/10.7195/ri14.v10i1.113

Sahm, M., Belleflamme, P., Lambert, T., \& Schwienbacher, A. (2014). Corrigendum to "crowdfunding: Tapping the right crowd". Journal of Business Venturing, 29(5), 610-611. doi:10.1016/j.jbusvent.2014.06.001

Sánchez-González, M., \& Palomo-Torres, M. (2014). Knowledge and assessment of crowdfunding in communication. The view of journalists and future journalists. Comunicar, 22(43), 101-110. doi: 10.3916/C43-2014-10

Sawers, P. (2014). The past, present and future of crowdfunding. Recuperado de http:// thenextweb.com/insider/2014/01/09/past-present-future-crowdfunding 


\section{MONOGRÁFICO}

Schwienbacher, A., \& Larralde, B. (2010). Crowdfunding of Small Entrepreneurial Ventures. Handbook of Entrepreneurial Finance, Oxford University Press. Recuperado de e http://dx.doi.org/10.2139/ssrn.1699183

Solera Navarro, F., \& Cabello Fernández-Delgado, F. (2015). Crowdfunding para la producción cultural basada en el procomún: el caso de Goteo (2011-2014). Historia y Comunicación Social, 20 (2), 447-464.

SpaceTec Capital Partners GmbH (2014). Crowdfunding innovative ventures in Europe: The financial ecosystem and regulatory landscape. A study prepared for the European Commission DG Communications Networks, Content \& Technology. ISBN 978-92-79-43849-3; doi: 10.2759/91398.

Stengel, G. (2014). Crowdfunding: Raise Money And Build Your Fan Base. Forbes. Recuperado de http://goo.gl/fPRxEi

Vitale, $M^{\text {a }}$ A. (2013): Êthos y legitimación política en los discursos de asunción de la presidente argentina Cristina Fernández de Kirchner. Icono 14, 11(1), 05-25. doi: 10.7195/ri14.v11i1.529

Wardrop, R., Zhang, B., Rau, R., \& Gray, M. (2015). Moving mainstream. The European Alternative Finance Benchmarking Report. Retrieved from www.scopus.com Willems, W. (2013). What characteristics of crowdfunding platforms influence the success rate? Master thesis. Cultural Economics \& Entrepreneurship, Erasmus Universiteit, Rotterdam.

Windle, R., \& Warren, S. (2014). Communication Skills. CADRE. Section 4. Recuperado de http://www.directionservice.org/cadre/section4.cfm

Wittmann, B. C., Daw, N. D., Seymour, B., \& Dolan, R. J. (2008). Striatal activity underlies novelty-based choice in humans. Neuron, 58(6), 967-973. doi:10.1016/j.neuron.2008.04.027

Zheng, H., Zhang, M., Xu, B., \& Wang, T. (2015). Improving sponsor's experience in reward-based crowdfunding: a psychological ownership persperctive. PACIS 2015 Proceedings. Paper 158. Recuperado de http://aisel.aisnet.org/pacis2015/158

\section{Agradecimientos}

Este artículo se inscribe en el proyecto financiado por el Ministerio de Economía y Competitividad (España) con referencia: EC02014-59688-R, Programa Estatal de Investigación, Desarrollo e Innovación Orientada a los Retos de la Sociedad, en el marco del Plan Estatal de Investigación Científica y Técnica y de Innovación 2013-2016. 